\title{
Crecimiento diamétrico de arboles caducifolios y perennifolios del bosque mesófilo de montaña en los alrededores de Xalapa
}

\author{
Guadalupe Williams-Linera ${ }^{1}$
}

\begin{abstract}
RESUMEN
El crecimiento diamétrico anual de los árboles es una variable esencial para eligir las especies que deben usarse para reforestación, en plantaciones, en proyectos de restauración ecológica, y aún para plantarse en jardines y calles. El objetivo del presente trabajo fue determinar las tasas de crecimiento en diámetro de algunas especies arbóreas caducifolias y perennifolias, dominantes en el bosque mesófilo de montaña de los alrededores de Xalapa, Veracruz. El crecimiento diamétrico anual de 29 especies arbóreas se midió durante cinco años en el bosque del Jardín Botánico Clavijero (112 individuos) y en el Parque Ecológico Clavijero (80 individuos), y durante un año en un bosque al norte de Banderilla (36 individuos). La tasa de crecimiento diamétrico de los árboles del bosque manejado en el Jardín Botánico $(0.83 \mathrm{~cm} /$ año $)$ fue significativamente mayor que la de los otros dos bosques naturales (0.29 cm/año), lo cual puede indicar el potencial de crecimiento de muchas especies. Además, la tasa de crecimiento diamétrico para el bosque natural es rápida en comparación con la estimada para otros bosques de montaña. Las especies con tasa de crecimiento más alta fueron Platanus mexicana, Quercus acutifolia, Cornus disciflora y Liquidambar styraciflua
\end{abstract}

var. mexicana en el Jardín Botánico, y $L$. styraciflua var. mexicana, Q. xalapensis, Clethra mexicana y $Q$. acutifolia en el bosque natural. La tasa de crecimiento diamétrico para las especies caducifolias

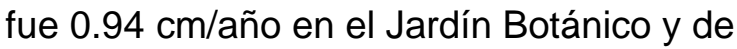

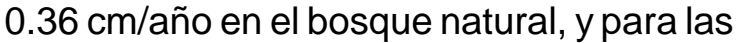
especies perennifolias fue 0.60 y 0.15 $\mathrm{cm} / a n ̃ o, ~ r e s p e c t i v a m e n t e$.

\section{PALABRAS CLAVE:}

Arboles caducifolios, árboles perennifolios, bosque mesófilo de montaña, crecimiento diamétrico, México, Veracruz.

\begin{abstract}
Diameter growth is an important variable to select tree species for plantations, reforestation and ecological restoration projects, and for planting in gardens and along avenues. The objective of this study was to determine diameter growth rates in both, deciduous and broadleaf evergreen tree species, in a lower montane tropical forest near Xalapa, Veracruz, Mexico. Diametric growth was measured annually during five years in the Botanical Garden forest (112 individuals) and in the Parque Ecológico Clavijero (80 individuals), and during a year in Banderilla (36 individuals). Average diameter growth increment was greater in the managed forest at the
\end{abstract}

1 Investigadora. Departamento de Ecología Vegetal. Instituto de Ecología, A.C. A pdo. Postal 63. 91000 Xalapa, Ver. México.

Manuscrito recibido para su publicación el 13 de Mayo de 1996 
Botanical Garden $(0.83 \mathrm{~cm} / \mathrm{yr})$ than in the natural forest $(0.29 \mathrm{~cm} / \mathrm{yr})$. This suggests forest tree species have a high potential to increment growth rates. Growth in diameter in the natural forest was faster comparing with other montane forests. Species with the highest growth rates were Platanus mexicana, Quercus acutifolia, Cornus disciflora and Liquidambar styraciflua var. mexicana in the Botanical Garden, and L. styraciflua var. mexicana, Q. xalapensis, Clethra mexicana and $Q$. acutifolia in the natural forest. Deciduous species diameter growth was $0.94 \mathrm{~cm} / \mathrm{yr}$ in the Botanical Garden and $0.36 \mathrm{~cm} / \mathrm{yr}$ in the natural forest, and broadleaf evergreen grew 0.60 and 0.15 $\mathrm{cm} / \mathrm{yr}$, respectively.

KEY WORDS:

Deciduous trees, evergreen trees, cloud forest, diameter growth rate, Mexico, Veracruz.

\section{INTRODUCCION}

Los bosques mesófilos de montaña (BMM) son importantes económica y ecológicamente. Estos bosques, a pesar de no estar sujetos a un uso racional y sostenible, son una fuente de ingresos para los habitantes locales que extraen de ellos vigas, tablas, carbón, leña y productos no maderables como plantas para venta, follaje de ornato, flores, medicinas, que contribuyen sustancialmente a la economía familiar. Desde un punto de vista ecológico, la presencia del bosque mantiene la captación de agua, conserva manantiales y mantos freáticos, evita la erosión del suelo y las inundaciones. Los bosques mesófilos, aunque ocupan sólo el $1 \%$ de la superficie del país, contribuyen con el $10 \%$ a la biodiversidad de México y por lo tanto son el tipo de vegetación más diverso (Rzedowski, 1992). De esta forma, el BMM es importante para mantener la biodiversidad local y contribuir a mantener la biodiversidad de la República Mexicana. En los alrededores de Xalapa, Veracruz, al igual que en todo México y Latinoamérica, el BMM está desapareciendo y actualmente se encuentra representado por algunos manchones que deben protegerse. Además, en esta región se deben buscar alternativas suplementarias a la conservación.

Frecuentemente es necesario conocer qué tan rápido crecen los árboles, ya sea para investigación o para planear su utilización. A menudo se utiliza la medición de los diámetros para evaluar a los tratamientos silviculturales sobre el crecimiento de los árboles del bosque. El crecimiento diamétrico medio anual varía considerablemente de un sitio a otro aún dentro de un mismo tipo de vegetación. Algunos factores que afectan la tasa de crecimiento de árboles individuales son la densidad, la edad, la vitalidad de los árboles del dosel superior, los cambios en los regímenes luminosos, el espacio para crecer, la disponibilidad de agua, la temperatura, la producción de semillas, la herbivoría y las enfermedades (Stoddard, 1968; Veillon, 1985).

El conocimiento básico sobre el crecimiento de los árboles es esencial para proponer el manejo de algunas especies de BMM. La información sobre la tasa de crecimiento en diámetro ayudaría a elegir las especies de árboles que deben plantarse en jardines y calles, usarse para reforestación, en plantaciones y en proyectos de restauración ecológica. Aunado a estudios sobre crecimiento diamétrico, deben realizarse estudios sobre aspectos silvícolas, ecológicos, y de utilización tanto de la madera como de productos no maderables. 


\section{OBJETIVO}

El objetivo del presente trabajo fue determinar y comparar las tasas de crecimiento en diámetro de algunas especies arbóreas caducifolias y perennifolias, dominantes en tres sitios representativos del bosque mesófilo de montaña en los alrededores de Xalapa, Veracruz.

\section{SITIOS DE ESTUDIO}

En la región de Xalapa, Veracruz, la precipitación anual media es alrededor de $1500 \mathrm{~mm}$ con una estación relativamente seca entre noviembre y abril, y la temperatura media es de $18^{\circ} \mathrm{C}$. En esta región se seleccionaron tres fragmentos de bosque mesófilo de montaña (bosque de niebla o bosque caducifolio). Un sitio de estudio fue el bosque del Jardín Botánico Clavijero y otro el área forestal protegida del Parque Ecológico Clavijero $\left(19^{\circ} 30^{\prime} \mathrm{N}, 96^{\circ}\right.$ $\left.57^{\prime} \mathrm{W}\right)$. Estos fragmentos de bosque se localizan en el km 2.5 de la carretera Xalapa-Coatepec; se encuentran separados por unos $300 \mathrm{~m}$, a una altitud de 1300 y $1250 \mathrm{~m}$, respectivamente. Otro sitio de estudio fue un bosque localizado a $1 \mathrm{~km}$ al norte de Banderilla, Veracruz $\left(19^{\circ} 35^{\prime} \mathrm{N}, 96^{\circ}\right.$ $75^{\prime} \mathrm{W}$ ), a una altitud de $1450 \mathrm{~m}$. Estos tres sitios son representativos del bosque mesófilo de montaña de la región y son parecidos entre sí tanto en estructura de la vegetación arbórea como en composición florística (Williams-Linera et al., 1996). Las especies seleccionadas para cada sitio se presentan en la Tabla 1, y la familia, nombre común y usos conocidos se presentan en el Apéndice.

\section{METODOLOGIA}

En los tres sitios de estudio se determinó el crecimiento diamétrico (cm/año) de árboles $\geq 5 \mathrm{~cm}$ DAP (diámetro a la altura del pecho a $1.3 \mathrm{~m})$. A cada individuo se le colocó un clavo con una etiqueta a $1.5 \mathrm{~m}$ del suelo, el cual sirvió de referencia para medir el DAP siempre a la misma altura utilizando cinta diamétrica. En el Jardín Botánico (JB) se seleccionaron 112 individuos que se midieron anualmente en agosto, de 1990 a 1994. En el Parque Ecológico se midieron 80 individuos en octubre, 1990 y se relocalizaron en octubre de 1993 y 1994. En Banderilla se eligieron 36 árboles y se midieron en octubre de 1993 y de 1994.

Las tasas de crecimiento diamétrico (cm/año) no presentaron una distribución normal, a pesar de esto, las medias se compararon mediante análisis de varianza (ANOVA) de una y dos-vias, ya que al utilizar métodos no paramétricos se obtuvieron los mismos resultados. Cuando se encontraron diferencias significativas se realizaron comparaciones múltiples (Prueba de Tukey, alfa =0.05; Daniel, 1983).

\section{RESULTADOS}

La tasa de crecimiento diamétrico fue significativamente mayor para el Jardín Botánico, seguida del Parque Ecológico y de Banderilla que no difirieron estadísticamente entre sí (ANOVA, dos vías, $\mathrm{P}<$ 0.01; Tabla 1). Los árboles caducifolios tuvieron una tasa media de crecimiento diamétrico más alta que los árboles perennifolios. Sin embargo, sólo las especies caducifolias del Jardín Botánico tuvieron un crecimiento significativamente mayor que las perennifolias y caducifolias de los otros dos sitios (ANOVA, $P=0.03$; Fig. 1).

La tasa de crecimiento no fue estadísticamente diferente entre las clases diamétricas (ANOVA dos vias, $\mathrm{P}=0.35$ ). Las clases más pequeñas tuvieron un 
Tabla 1. Crecimiento diamétrico ( $\mathrm{cm} / \mathrm{año}$ ) de especies arbóreas del bosque de neblina en los alrededores de Xalapa, Veracruz.

Se presentan resultados por clases diamétricas; entre paréntesis se encuentra el número de individuos promediados. Los números en negritas representan el crecimiento diamétrico medio de cada especies en el sitio. ${ }^{1}$ indica que la especie es caducifolia $y^{2}$ que es perennifolia.

\begin{tabular}{|c|c|c|c|c|}
\hline ESPECIES & $\begin{array}{l}\text { CLASE } \\
(\mathrm{cm})\end{array}$ & $\begin{array}{l}\text { JARDIN } \\
\text { BOTANICO }\end{array}$ & $\begin{array}{l}\text { PARQUE } \\
\text { ECOLOGICO }\end{array}$ & BANDERILLA \\
\hline \multirow[t]{2}{*}{ 1. Carpinus caroliniana ${ }^{1}$} & $\begin{array}{c}5-9.9 \\
10-19.9 \\
20-29.9\end{array}$ & $\begin{array}{l}0.79 \\
0.68(2) \\
0.50(2) \\
1.53(1) \\
--\end{array}$ & $\begin{array}{l}\mathbf{0 . 1 8} \\
0.20(14) \\
0.11(8) \\
0.29(2) \\
0.25(1)\end{array}$ & $\begin{array}{l}\mathbf{0 . 0 4} \\
0.07(3) \\
0.00(2) \\
-- \\
--\end{array}$ \\
\hline & $\begin{array}{l}30-39.9 \\
40-49.9 \\
50-59.9\end{array}$ & $\begin{array}{l}0.41(2) \\
1.21(2)\end{array}$ & $\begin{array}{l}-- \\
--\end{array}$ & $\begin{array}{l}-- \\
--\end{array}$ \\
\hline 2. Cinnamomum barbeyanum ${ }^{2}$ & $\begin{array}{c}5-9.9 \\
10-19.9\end{array}$ & $\begin{array}{l}\mathbf{0 . 6 3} \\
\overline{--} \\
0.63(2)\end{array}$ & $\begin{array}{l}0.21 \\
0.16(5) \\
0.50(1)\end{array}$ & $\begin{array}{l}-- \\
-- \\
--\end{array}$ \\
\hline 3. Clethra mexicana ${ }^{1}$ & $\begin{array}{c}5-9.9 \\
10-19.9 \\
20-29.9 \\
30-39.9 \\
40-49.9 \\
50-59.9 \\
60-69.9\end{array}$ & $\begin{array}{l}0.61 \\
-- \\
0.68(3) \\
0.98(3) \\
-- \\
0.48(1) \\
0.05(1) \\
0.41(3)\end{array}$ & $\begin{array}{l}0.34 \\
0.03(1) \\
-- \\
-- \\
0.45(3) \\
-- \\
-- \\
--\end{array}$ & $\begin{array}{l}\mathbf{0 . 3 0} \\
0.00(1) \\
0.60(2) \\
0.00(1) \\
-- \\
-- \\
--\end{array}$ \\
\hline 4. Cornus disciflora ${ }^{1}$ & $\begin{array}{l}5-9.9 \\
10-19.9\end{array}$ & $\begin{array}{l}\mathbf{1 . 7 3} \\
1.50(3) \\
2.08(2)\end{array}$ & $\begin{array}{l}-- \\
-- \\
--\end{array}$ & $\begin{array}{l}-- \\
-- \\
--\end{array}$ \\
\hline 5. Dendropanax arboreus ${ }^{2}$ & $10-19.9$ & $\begin{array}{l}1.58 \\
1.58(1)\end{array}$ & $\begin{array}{l}-- \\
--\end{array}$ & $\begin{array}{l}-- \\
--\end{array}$ \\
\hline 6. Eugenia mexicana ${ }^{2}$ & 5- 9.9 & -- & $\begin{array}{l}0.13 \\
0.13(4)\end{array}$ & -- \\
\hline 7. Eugenia xalapensis ${ }^{2}$ & 5- 9.9 & $\begin{array}{l}-- \\
--\end{array}$ & $\begin{array}{l}0.10 \\
0.10(1)\end{array}$ & $\begin{array}{l}-- \\
--\end{array}$ \\
\hline 8. Hedyosmum mexicanum ${ }^{2}$ & $\begin{array}{c}5-9.9 \\
10-19.9\end{array}$ & $\begin{array}{l}\mathbf{0 . 4 7} \\
0.45(2) \\
0.50(1)\end{array}$ & $\begin{array}{l}-- \\
-- \\
--\end{array}$ & $\begin{array}{l}\mathbf{0 . 0 0} \\
0.00(2) \\
0.00(1)\end{array}$ \\
\hline 9. Ilex tolucana ${ }^{2}$ & $\begin{array}{c}5-9.9 \\
10-19.9 \\
20-29.9\end{array}$ & $\begin{array}{l}0.31 \\
0.13(1) \\
0.00(1) \\
0.55(2)\end{array}$ & $\begin{array}{l}0.10 \\
0.10(1) \\
-- \\
--\end{array}$ & $\begin{array}{l}-- \\
-- \\
-- \\
--\end{array}$ \\
\hline 10. Lippia myriocephala² & $20-29.9$ & $\begin{array}{l}0.60 \\
0.60(1)\end{array}$ & $\begin{array}{l}-- \\
--\end{array}$ & $\begin{array}{l}-- \\
--\end{array}$ \\
\hline
\end{tabular}


... cont Tabla 1. Crecimiento diamétrico ...

\section{ESPECIES}

10. Liquidambar styraciflua'

11. Magnolia schideana ${ }^{2}$

12. Matudae trinervia ${ }^{2}$

13. Meliosma alba ${ }^{1}$

14. Oreopanax capitatus ${ }^{2}$

15. Oreopanax xalapensis ${ }^{2}$

16. Platanus mexicana ${ }^{2}$

17. Quercus acutifolia ${ }^{2}$

18. Quercus germana²

CLASE
$(\mathrm{cm})$

JARDIN

BOTANICO

PARQUE
ECOLOGICO

BANDERILLA

\subsection{6}

5- 9.9

10-19.9

20-29.9

30-39.9

$40-49.9$

50-59.9

60-69.9

$>70$

$1 . \overline{30}(1)$

$1.16(7)$

$0.10(1)$

$--$

$0.10(1)$

5- 9.9

10-19.9

0.98

$0.98(1)$

$--$

$5-9.9$
$10-19.9$
$30-39.9$
$>\quad 70$

20-29.9

1.10

1.10 (1)

0.46

0.28 (1)

$0.53(1)$

0.58 (1)

0.86

$\begin{array}{ll} & 0.86(2) \\ & \mathbf{0 . 7 5}\end{array}$

5- 9.9

0.75 (1)

10-10.9

20-29.9

30-39.9

$>70$

1.94

1.80 (1)

2.15 (1)

2.56 (2)

0.63 (1)

1.47

--

$-$

$0 . \overline{2}$

0.20 (1)

2.04 (4)

2.45 (1)

1.06 (2)

0.33 (1)

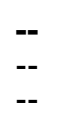

0.16

0.14 (2)

0.20 (1)

$\begin{array}{ll}-- & - \\ -- & - \\ -- & -- \\ -- & - \\ -- & - \\ -- & - \\ -- & - \\ -- & -\end{array}$

0.13

0.13 (4)

$\mathbf{0 . 0 0}$

0.00 (5)

$\begin{array}{ll}-- & - \\ -- & - \\ -- & - \\ -- & --\end{array}$

10-19.9

20-29.9

30-39.9

40-49.9

50-59.9

1.93

1.37 (3)

$2.68(3)$

$2.10(1)$

1.58 (2)

0.26

0.13 (1)

0.40 (1)

--

$--$

\subsection{6}

5- 9.9

10-19.9

20-29.9

30-39.9

40-49.9

50-59.9

60-69.9

0.16
--
--
--
$0.33(1)$
$0.11(2)$
$0.15(2)$
$0.38(1)$
$0.02(2)$
0.00 (1)

0.15 (4)

--

$-$

$-$
--
--
--
--

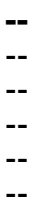

0.17

0.61 (1)

0.07 (2)

0.08 (1)

$0.03(1)$

0.02 (2)

$--$ 
... cont Tabla 1. Crecimiento diamétrico ..

\section{ESPECIES}

20. Quercus insignis ${ }^{1}$

21. Quercus leioplhylla

22. Quercus xalapensis

23. Rapanea myricoides ${ }^{1}$

24. Saurauia belizensis ${ }^{2}$

25. Styrax glabrescens ${ }^{2}$

26. Symplocos coccinea $a^{2}$

27. Trema micrantha ${ }^{2}$

28. Trichilia havanensis ${ }^{2}$

29. Turpinia insignis ${ }^{2}$

\section{CLASE}

$(\mathrm{cm})$

$$
\text { 5- } 9.9
$$
40-49.9 $>70$

5- 9.9 20-29.9 30-39.9

50-59.9 60-69.9

$>70$

$\begin{array}{ll} & 0.62 \\ 5-9.9 & 0.50(1) \\ 10-19.9 & -- \\ 20-29.9 & 1.20(1) \\ 30-39.9 & -- \\ 40-49.9 & -- \\ 60-69.9 & -- \\ >70 & 0.47(3)\end{array}$

5- 9.9 10-19.9

$10-19.9$

30.-39.9

5- 9.9

20-29.9

5- 9.9

5- 9.9

10-19.9

20-29.9 $\mathbf{0 . 7 5}$

JARDIN BOTANICO

\subsection{9}

$0.03(1)$

$0.75(1)$

0.66

$0.53(1)$

0.00 (1)

$0.95(1)$

$0.78(1)$

0.75 (4)

$0.53(1)$

0.78 (8)

0.15

0.15 (1)

0.05

0.15 (1)

0.51

0.51 (2)

1.18

1.18 (1)

0.21

0.21 (2)

\subsection{1}

0.66 (1)

$0.06(2)$

0.08 (1)
PARQUE ECOLOGICO

0.00

0.00 (1)
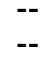

0.05

$0.05(2)$

$0.50(1)$

--

--

$--$

\subsection{3}

$0.00(1)$

0.47 (3)

$0 . \overline{50}(1)$

$0.55(1)$

0.58 (1)

$--$

$--$

$--$

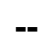

$--$

$--$

$--$

--

$\overline{--}$

$--$

0.20

$0.18(6)$

$0.24(3)$

--

0.35
BANDERILLA

-
--
--
-
-
-
-
-
-
-
--

0.20

0.10 (2)

0.30 (2)

$-$

$-$

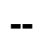

$-$

$-$

$-$

--

--

--

$--$

$-$

$--$

--

0.28

0.25 (4)

0.40 (1)

PROMEDIO

0.83

0.14 


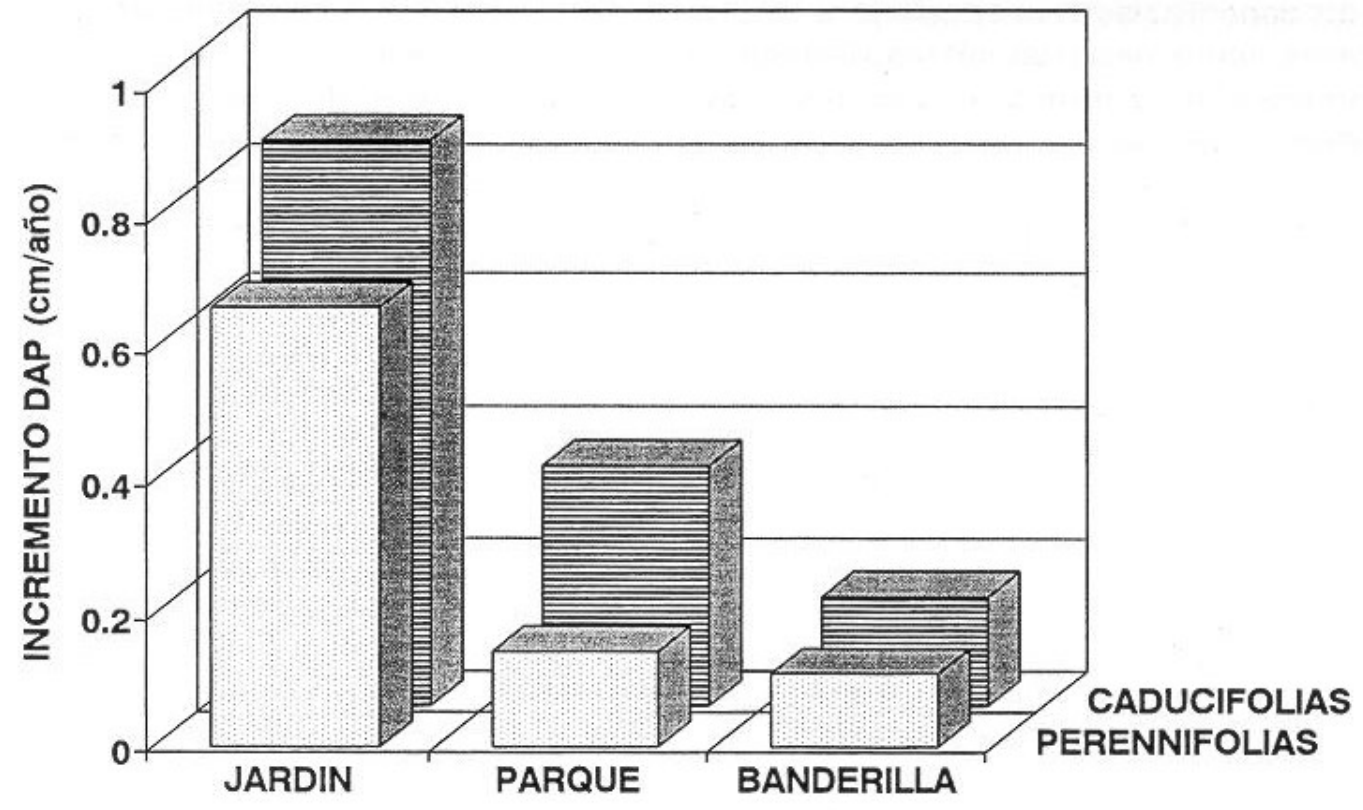

Figura 1. Incremento diamétrico promedio (cm/año) de las especies caducifolias y perennifolias medidas en los tres sitios de estudio: Jardín Botánico Clavijero, Parque Ecológico Clavijero y bosque cerca de Banderilla.

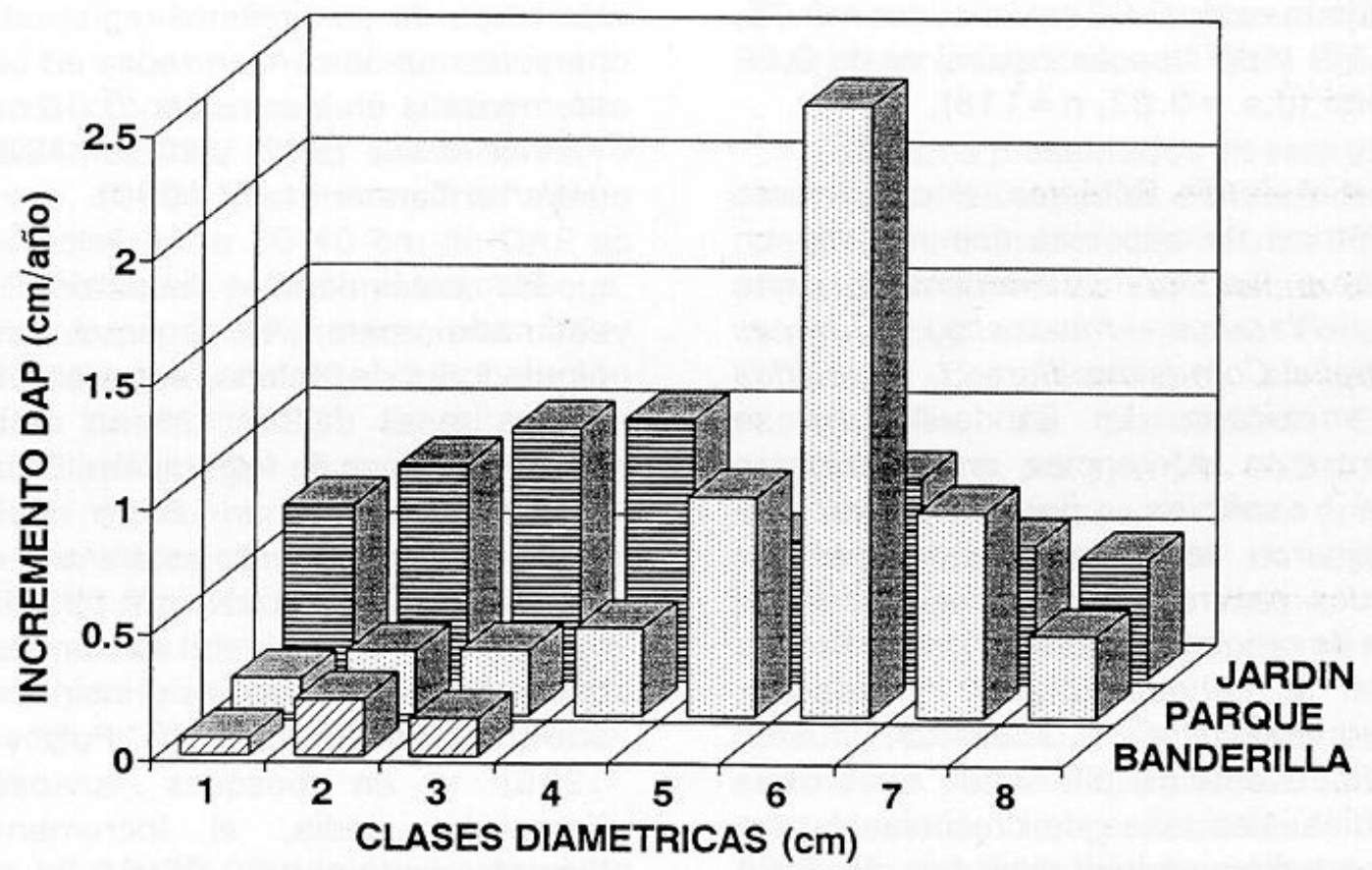

Figura 2. Incremento diamétrico promedio ( $\mathrm{cm} / a n ̃ o)$ en los tres sitios de estudio: J ardín Botánico Clavijero, Parque Ecológico Clavijero y bosque cerca de Banderilla. Las clases 1 a 8 corresponden a los intervalos 5-9.9,10-19.9, 20-29.9, 30-39.9, 40-49.9, 50-59.9, 6069.9 y > $70 \mathrm{~cm}$ de DAP, respectivamente. 
crecimiento relativamente bajo, el cual se incrementó hacia las clases diamétricas intermedias y disminuyó para las clases diamétricas mayores que presumiblemente contienen a los árboles más viejos (Fig. 2 ). En el Parque Ecológico, la clase 6 es un dato aislado ("outlier") ya que se trata de un sólo individuo de Liquidambar styraciflua var. mexicana con un crecimiento no representativo. En el bosque de Banderilla, las bajas tasas de crecimiento se deben a que los individuos marcados pertenecen a las clases diamétricas menores (Fig. 2).

El J B representa un bosque manejado y las especies son favorecidas y cuidadas, y algunos individuos fueron plantados, lo cual puede explicar su alta tasa de crecimiento diamétrico. Los otros dos sitios son estadísticamente similares entre sí, y representan el bosque natural de la región. A partir de lo anterior se considera que la tasa de crecimiento del bosque manejado es de $0.83 \mathrm{~cm} /$ año (d.s. $=0.73$, $\mathrm{n}=112$ ) y del bosque natural es de 0.29 $\mathrm{cm} /$ año (d.s. $=0.52, \mathrm{n}=116$ ).

En el J ardín Botánico, el crecimiento diamétrico de especies con más de un individuo, fue significativamente más alto para Platanus mexicana, Quercus acutifolia, Cornus disciflora y $L$. styraciflua var. mexicana. En Banderilla, no se encontraron diferencias en crecimiento entre especies, pero cuando se combinaron las especies de los dos bosques naturales, las especies con las tasas de crecimiento diamétrico más altas fueron L. styraciflua, Q. xalapensis, Clethra mexicana y $Q$. acutifolia, y fueron significativamente diferentes a las otras especies. Las tasas de crecimiento para las especies caducifolias fue de 0.94 cm/año en el J ardín Botánico y de 0.36

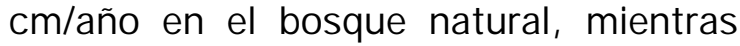
que para las especies perennifolias fue 0.60 y $0.15 \mathrm{~cm} / a n ̃ o$, respectivamente.

\section{DISCUSION}

La tasa de crecimiento diamétrico medio para el bosque del J ardín Botánico fue más del doble de cualquiera estimada para bosques de montaña. Las tasas de crecimiento del bosques natural $(0.29$ $\mathrm{cm}$ /año) fue similar a las de otros bosques de montaña. En las Montañas Luquillo, Puerto Rico, en el bosque montano bajo muy húmedo, el crecimiento diamétrico

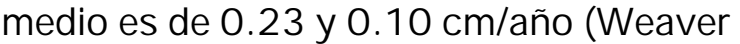
y Murphy, 1990). En Venezuela, el promedio de crecimiento anual para el bosque húmedo montano bajo (1500 a $2500 \mathrm{~m}$ de altitud) es de $0.20 \mathrm{~cm} /$ año, y para el bosque húmedo premontano (1000 a $1500 \mathrm{~m}$ ) es de $0.25 \mathrm{~cm} /$ año (Veillon, 1985). En Australia, en un bosque tropical lluvioso de montaña, el crecimiento diamétrico medio es 0.27

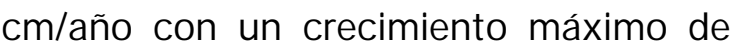

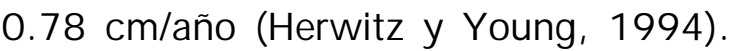
Sin embargo, en el bosque natural de Xalapa y Banderilla, las tasas de crecimiento registradas son mayores que las encontradas en bosques de montaña

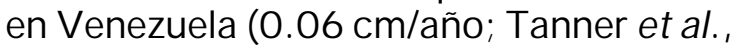
1992) y en Jamaica $(0.02$ cm/año; Tanner et al., 1990).

El crecimiento diamétrico anual estimado para el bosque en los alrededores de Xalapa, entra en el rango de las tasas de crecimiento estimadas para otros tipos de vegetación. En la selva tropical húmeda en Barro Colorado, Panamá el crecimiento está entre < 0.01 a $1.5 \mathrm{~cm} /$ año (Lang y Knight 1983), en un manglar en Malasia, el crecimiento en diámetro de las especies principales varía

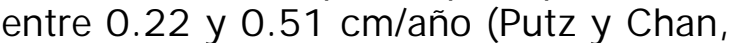
1986) y en bosques lluviosos en Karnataka, India, el incremento en diámetro varía entre 0.25 a $0.31 \mathrm{~cm} / a n ̃ o$ (Rai y Proctor, 1986).

En el BMM, el crecimiento en diámetro fue muy variable tanto entre especies 
como entre clases diamétricas. La tasa de crecimiento más pequeña fue $<0.01$

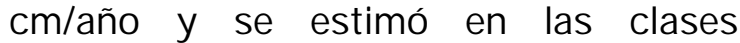
diamétricas $<30 \mathrm{~cm}$ DAP, aunque algunos árboles grandes ( $>30 \mathrm{~cm} \mathrm{DAP)}$ tuvieron un crecimiento muy pequeño. Veillon (1985) considera que el crecimiento medio de las diferentes clases diamétricas presenta una variedad de casos y que no hay una regla general. Frecuentemente el crecimiento diamétrico es menor en la primera clase, que contiene muchos árboles suprimidos 0 dominados y, en la última clase, la de los gruesos, muchos de ellos seniles y estancados en su desarrollo. Pero también se destacan sitios donde los árboles más gruesos son los que crecen más.

El incremento en circunferencia continua durante toda la vida del árbol, aunque declina con la senilidad. Sin embargo, hay registros de árboles tropicales saludables que no presentan crecimiento diamétrico por periodos largos. En varios lugares se han estimado tasas de crecimiento nulo y muy lento (UNESCO, 1978). En la selva de Barro Colorado, Panamá, el incremento medio para árboles entre $30-40 \mathrm{~cm}$ de DAP es de $0.90 \mathrm{~cm} / a n ̃ o$, pero en el sotobosque, los árboles pequeños crecieron muy lentamente, generalmente $<0.1 \mathrm{~cm} / a n ̃ o$ (Lang y Knight, 1983). En el bosque tropical lluvioso del Amazonas, se ha estimado que un árbol promedio en la clase diamétrica $2535 \mathrm{~cm}$ tiene un incremento diamétrico de $0.80 \mathrm{~cm} / a n ̃ o, y$ en las clases de 25 a $155 \mathrm{~cm}$, el incremento sería entre 0.37 y $0.8 \mathrm{~cm} / a n ̃ o$ (Heinsdijk, 1963, citado en UNESCO, 1978).

En el Jardín Botánico la tasa de

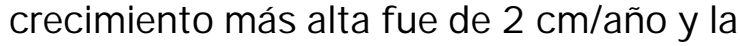
alcanzaron varios individuos jóvenes de Platanus mexicana ( $<40 \mathrm{~cm} \mathrm{DAP)} \mathrm{y} \mathrm{de}$ Cornus disciflora ( $<20 \mathrm{~cm} \mathrm{DAP)}$, aunque también la tuvieron algunos individuos maduros de Quercus acutifolia. En el bosque natural sólo individuos maduros (40 a $60 \mathrm{~cm}$ DAP) de Liquidambar styraciflua var. mexicana alcanzaron esta tasa de crecimiento.

En general, las especies de BMM con crecimiento más altos fueron Liquidambar styraciflua var. mexicana y Platanus mexicana. En los Estados Unidos, especies muy cercanas presentan tasas de crecimiento diamétrico similares a las especies que crecen en México. El crecimiento diamétrico de Liquidambar styraciflua para individuos maduros es de

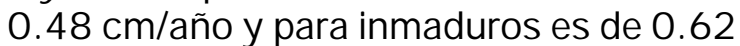

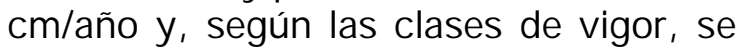
menciona que los individuos en clases altas alcanzan $1.01 \mathrm{~cm} / a n ̃ o$, en medias

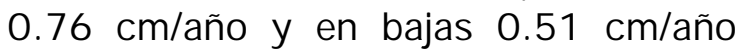
(Fowells, 1965). El mismo autor señala que Platanus occidentalis, considerado como un árbol de crecimiento rápido durante toda su vida, alcanza una tasa de crecimiento diamétrico de $0.91 \mathrm{~cm} / a n ̃ o$.

Los datos presentados en este estudio indican que las especies arbóreas de BM M tienen un crecimiento diamétrico alto $\mathrm{y}$, la tasa de crecimiento del bosque del J B, sugiere que estas especies tienen un potencial de crecimiento elevado en condiciones de plantación. Existen algunos casos, por ejemplo, en Indonesia, en suelos volcánicos fértiles, donde el potencial de crecimiento de especies arbóreas en condiciones más o menos controladas de densidad en una plantación es mucho mayor que en el bosque primario (UNESCO, 1978). Una silvicultura adecuada podría seguramente aumentar notablemente la productividad de los bosques naturales, sin necesidad de optar por su eliminación y sustitución por plantaciones de unas pocas especies autóctonas o exóticas (Veillon, 1985). 


\section{CONCLUSIONES}

La tasa de crecimiento diamétrico en el bosque del J B es 2.8 veces mayor que la estimada para el bosque natural, lo cual puede indicar el potencial de crecimiento de muchas especies. Además, la tasa de crecimiento diamétrico para el BMM natural es alta en comparación con la estimada para otros bosques de montaña.

Las especies de mayor crecimiento diamétrico son Platanus mexicana, Quercus acutifolia, Cornus disciflora y Liquidambar macrophylla en el J ardín Botánico, y L. styraciflua, Q. xalapensis, Clethra mexicana y $Q$. acutifolia en el bosque natural. Las especie caducifolias que presentaron una tasa de crecimiento diamétrico mayor, además, son los árboles dominantes del dosel superior del bosque, en cambio, las perennifolias tuvieron un crecimiento menor, se encuentran en el sotobosque y no llegan a formar parte del dosel superior.

En restauración ecológica, recreación de bosques, o en planes innovadores para plantar árboles primarios de BMM en avenidas o jardines, se deben incluir tanto árboles caducifolios como perennifolios que complementan la fisonomía de bosque mesófilo de montaña de los alrededores de Xalapa, Veracruz. Para dichos proyectos hace falta más información sobre las tasas de crecimiento tanto diamétrico como en altura de las diferentes especies arbóreas en distintas condiciones ambientales.

\section{RECONOCIMIENTOS}

Agradezco al Biol. J avier Tolome su ayuda en la toma de datos y a dos revisores anónimos sus valiosos comentarios al manuscrito. El presente estudio se llevó a cabo con el apoyo del proyecto CONACyT Ref:4334-N, responsable $\mathrm{G}$. Williams-Linera.

\section{REFERENCIAS}

Barajas, J. 1980. Anatomía de maderas de México No. 3; diez especies de bosque caducifolio de las cercanías de Xalapa, Ver. México. Biótica 5(1):2340.

Carmona, T. F. 1979. Características histológicas de la madera de cuatro especies del bosque caducifolio de México. Tesis de Licenciatura. Facultad de Ciencias. UNAM. México, D. F.

Daniel, W. W. 1983. Biostatistics: a foundation for analysis in the health sciences. J ohn Wiley \& Sons. Nueva York. 534 p.

Fowells, H. A. 1965. Silvics of forest trees of the United States. US Department of Agriculture. Forest Service. Agriculture Handbook No. 271. Washington, DC.

Herwitz, S. R. y S. S. Young. 1994. Mortality, recruitment, and grow th rates of montane tropical rain forest canopy trees on M ount Bellenden-Ker, Northeast Queensland, Australia. Biotropica 26(4):350-361.

Lang, G. E. y D. H. Knight. 1983. Tree growth, mortality recruitment and canopy gap formation during a 10 year period in a tropical moist forest. Ecology 64(5):1075-1080.

Ortega, R. V. 1987. Los árboles silvestres más comunes en el J ardín Botánico Francisco J. Clavijero, Xalapa, Veracruz. Cuaderno de Divulgación No. 34. INIREB, Xalapa, Ver. 91 p.

Pennington, T. D. y J . Sarukhán K. 1968. Manual para la identificación de campo de los principales árboles 
tropicales de México. INIF-ONU-FAO. México, D. F. 413 p.

Putz, F. E. y H. T. Chan. 1986. Tree grow th, dynamics, and productivity in a mature mangrove forest in Malaysia. For. Ecol. Manag. 17(2-3):211-230.

Rai, S. N. y J . Proctor. 1986. Ecological studies on four rainforests in Karnataka, India. J. Ecol. 74(2):439454.

Rzedowski, J. 1992. Diversidad y orígenes de la flora fanerogámica de México". In: G. Halffter, ed. La diversidad biológica de Iberoamérica. Acta Zoológica Mexicana-CYTED. Xalapa, Ver. pp: 313-335.

Sosa, V. 1976. Hammamelidaceae de Veracruz. Biótica 1(1):45-59.

Stoddard, C. H. 1968. Essentials of forestry practice. The Ronald Press Company. Nueva York. 362 p.

Tanner, E. V. J ., V. Kapos, S. Freskos, J . R. Healey y A. M. Theobald. 1990. Nitrogen and phosphorus fertilization of J amaican montane forest trees. J . Trop. Ecol. 6(2):231-238.

Tanner, E. V. J., V. Kapos y W. Franco. 1992. Nitrogen and phosphorus fertilization effects on Venezuelan montane forest trunk growth and litterfall. Ecology 73(1):78-86.

UNESCO. 1978. Tropical forest ecosystems. A state of knowledge report. UNESCO- UNEP. Paris. 683 p.

Veillon, J. P. 1985. El crecimiento de algunos bosques naturales de Venezuela en relación con los parámetros del medio ambiente. Rev. For. Venez. 19(1):5-120.
Weaver, P. L. y P. G. Murphy. 1990. Forest structure and productivity in Puerto Rico's Luquillo Mountains. Biotropica 22(1):69-82.

Williams-Linera, G., I. Pérez y J. Tolome. 1996. El bosque mesófilo de montaña y un gradiente altitudinal en el centro de Veracruz, México. La Ciencia y el Hombre 22. (En prensa).

\section{APENDICE}

Listado de especies arbóreas seleccionadas para el presente estudio. Se incluye la familia, el nombre común entre paréntesis y algunos usos.

1. Carpinus caroliniana Walt. BETULACEAE (pepinque). Es un árbol muy abundante y se encuentra en el estrato bajo del bosque. Su uso actual es leña y postes para cercas. Sin embargo, Carmona (1979) considera que su madera es susceptible de ser considerada como madera comercial y ser empleada en la fabricación de implementos agrícolas, muebles rurales, capas interiores de madera contrachapada y artículos torneados.

2. Cinnamomum aff. barbeyanum (Mez) Kosterm. LAURA CEAE (no se conoce el nombre común). Esta especie ha sido mal identificada como una Nectandra. No se conocen usos actuales 0 potenciales.

3. Clethra mexicana DC. CLETHRACEAE (marangola). Este árbol es abundamente en la región y forma parte del dosel superior del bosque; se usa para leña y carbón; ocasionalmente para la construcción de casas. Existen estudios sobre anatomía de esta madera (ver Barajas, 1980). 
4. Cornus disciflora Moc. \& Sessé ex DC. Prodr. CORNACEAE (aceitunillo). No se conocen usos importantes, podría utilizarse en reforestación para calles y jardines.

5. Dendropanax arboreus (L.) Planch. $\&$ Decne. ARALIACEAE (cucharo). Su madera tiene buenas características físicas para la fabricación de chapa, y por su bella apariencia podría usarse en un futuro para las caras de vista de madera terciada (Pennington y Sarukhán, 1968).

6. Eugenia mexicana Steudel MYRTACEAE (huesillo, escobilla real). No tiene usos conocidos pero debido a su follaje oscuro y brillante podría utilizarse como ornamental.

7. Eugenia xalapensis (Kunth) DC. MYRTACEAE (escobilla). Ejemplar tipo colectado en Xalapa, Ver. No tiene usos conocidos pero podría utilizarse como ornamental.

8. Hedyosmum mexicanum Cordemoy CHLORA NTHACEAE (platanillo, palo de agua). No se conocen usos importantes de esta especie. Podría utilizarse como ornamental.

9. Ilex tolucana Hemsley AQUIFOLIACEAE (palo prieto). Se utiliza únicamente para leña y carbón.

10. Lippia myriocephala Schlechtendal \& Cham. VERBENACEAE (palo gusano). Se utiliza únicamente para leña y carbón.

11. Liquidambar styraciflua L. var, m e x i c a n a e r s t ed. HAMAMELIDACEAE (ocozote, liquidambar). Es un árbol muy abundante y dominante en los bosques de la región. Se utiliza el bálsamo que se obtiene de incisiones en la corteza del tronco, se le atribuyen propiedades estimulantes, sudoríficas y estomacales. Se utilizó como saborizante del tabaco, incienso y en la elaboración de unguentos y emplastos. La madera es bastante apreciada, se utiliza para hacer tablas, toneles, chapas, herramientas y otros productos. Este árbol también se utiliza como ornamental en jardines y avenidas (Sosa, 1976).

12. Magnolia schiedeana Schlecht. MAGNOLIACEAE (magnolia). En Veracruz se cultiva en forma escasa como planta de ornato, en casas y jardines.

13. Matudaea trinervia Lund. HAM AM ELIDACEAE (guayabillo). Es un árbol abundante en el BMM de Chiapas. Se encontró plantado en el Jardín Botánico y podría utilizarse como ornamental.

14. Meliosma alba (Schlechtendal) Walp. SABIACEAE (palo blanco). La madera se utiliza en construcciones rurales y ocasionalmente en carpintería (Ortega, 1987).

15. Oreopanax capitatus (J acq.) Decne. $\&$ Planchon. ARA LIACEAE (cabellera de palo, coamatl, choco). Es un árbol hemiepífito, sus hojas se utilizan para envolver tamales.

16. Oreopanax xalapensis (H. B. \& K.) Decne. \& Planchon ARALIACEAE (mano de león, pata de gallo, siete hojas, tamalcoabite de montaña). El ejemplar tipo fue colectado en Xalapa, Ver. No se conocen usos importantes de esta planta, pero tiene buenas cualidades para considerarse como un árbol 0 arbusto de ornato (Ortega, 1987). 
17. Platanus mexicana Moric. PLATANACEAE (haya). No hay datos de usos industriales de la madera de esta especie. El árbol se usa como sombra o para obtener tablas para la construcción de casas (Pennington y Sarukhán, 1968). La madera se usa en carpintería para hacer platos y cucharas, también se dice que la hoja hervida sirve para el asma (Ortega, 1987). Existen estudios sobre anatomía de esta madera (ver Barajas, 1980).

18. Quercus acutifolia Nee. FAGACEAE (encino duela). Maderable y medicinal.

19. Quercus germana Cham. \& Schlechtendal FAGACEAE (roble, encino blanco). Maderable $y$ medicinal.

20. Quercus insignis Mart. \& Gal. FAGACEAE (encino avellano). En Jalisco, los frutos son empleados como alimento para cerdos.

21. Quercus leiophylla A. DC. FAGACEAE (encino de hoja menuda). Maderable.

22. Quercus xalapensis Humb. \& Bonpl. FAGACEAE (encino rojo, encino roble). Medicinal y maderable.

23. Rapanea myricoides (Schlechtendal) Lundell MYRSINACEAE (cerilla). Sólo se utiliza para leña y carbón, ocasionalmente para cercas.

24. Saurauia belizensis L. ACTINIDIACEAE (no se conoce el nombre común). Existen estudios sobre anatomía de esta madera (ver Barajas, 1980).

25. Styrax glabrescens Benth. STYRACACEAE (sajarillo, azahar de monte, jazmín). No se conocen usos importantes, pero podría ser utilizado en parques, jardines $y$ calles.

26. Symplocos coccinea Humb. \& Bonp. SYM PLOCACEAE (limoncillo). Es un árbol notable por su floración vistosa, podría ser utilizado para ornato en jardines y parques.

27. Trema micrantha (L.) Blume ULMACEAE (capulín, ixpepe). Su madera no recibe ningún uso industrial; la corteza fibrosa se usa para amarrar diversos objetos (Pennington y Sarukhán, 1968). En algunos sitios se emplea para la fabricación de papel amate.

28. Trichilia havanensis Jacq. MELIACEAE (rama tinaja). Su usa localmente para la fabricación de mangos de herramientas. Durante la celebración del Día de Muertos, se utiliza el follaje para adornar los altares. De las semillas se extrae un aceite que se usa en preparaciones para el pelo.

29. Turpinia insignis (H. B. \& K.) Tul. STAPHY LEACEAE (huevo de gato). Localmente se utiliza para leña y carbón; sin embargo, podría utilizarse como planta de ornato en jardines y parques, por su corta estatura, su floración y su follaje siempre verde y lustroso (Ortega, 1987). 